\title{
Pacific
}

Journal of

Mathematics

ON FACTOR REPRESENTATIONS OF DISCRETE RATIONAL NILPOTENT GROUPS AND THE PLANCHEREL FORMULA

LAWRENCE JAY CORWIN AND CAROLYN PFEFFER JOHNSTON 


\section{ON FACTOR REPRESENTATIONS \\ OF DISCRETE RATIONAL NILPOTENT GROUPS AND THE PLANCHEREL FORMULA}

\section{Lawrence Corwin and Carolyn Pfeffer Johnston}

The purpose of this paper is to extend the Kirillov orbit picture of representation theory for nilpotent Lie groups to discrete groups $G_{\mathbf{Q}}$ defined over the rationals $Q$, following a program begun by Roger Howe. Let $\mathrm{Ad}^{*}$ be the coadjoint action of $G_{Q}$ on the Pontryagin dual $\widehat{g_{Q}}$ of the Lie algebra of $G_{Q}$. It is shown that each coadjoint orbit closure is a coset of the annihilator of an ideal of $g_{Q}$, that a certain induced representation canonically associated with an orbit closure is a traceable factor, and that there is an orbital integral formula which gives the trace. Finally, a Plancherel formula is proved.

1. Introduction. In what follows, let $\mathbf{g}_{\mathbf{Q}}$ be a nilpotent Lie algebra with rational structure constants; i.e., $\mathbf{g}_{\mathbf{Q}}$ has a basis $\left\{X_{1}, \ldots, X_{n}\right\}$ of vectors such that

$$
\left[X_{i}, X_{j}\right]=\sum_{k=1}^{n} \alpha_{i, j, k} X_{k},
$$

with all $\alpha_{i, j, k} \in \mathbf{Q}$. Then we form a nilpotent group $G_{\mathbf{Q}}$ with base set $\mathbf{g}_{\mathbf{Q}}$, using the Campbell-Baker-Hausdorff formula to define a polynomial group multiplication, and we have a map $\exp : \mathbf{g}_{\mathbf{Q}} \rightarrow G_{\mathbf{Q}}$, $\exp \equiv$ Id on the set $\mathbf{g}_{\mathbf{Q}}$. We will think of $\mathbf{g}_{\mathbf{Q}}$ and $G_{\mathbf{Q}}$ as having the discrete topology. $G_{\mathbf{Q}}$ has no normal abelian group of finite index; therefore $G_{\mathbf{Q}}$ is not of Type I ([Tho]). The purpose of this paper is to develop an extension of the Kirillov orbit picture of representation theory for nilpotent Lie groups to discrete groups $G_{\mathbf{Q}}$ defined over the rationals, following a program begun by Roger Howe ([How1], [How2], [How3]). In particular, in [How2], he constructs an extension of the Kirillov theory to finitely generated discrete nilpotent groups without torsion; this work was a chief source of inspiration to us.

Although it is in general difficult to work with non-type-I groups (and in particular discrete groups lack some of the structure that nontype-I connected Lie groups $G$ possess: see [Puk], [Gdt]), it is possible to say a great deal in the present case. Let $\widehat{\mathbf{g}_{\mathbf{Q}}}$ denote the Pontryagin dual of the Lie algebra $\mathbf{g}_{\mathbf{Q}}$; we examine the structure of closures of 
orbits under the coadjoint action of $G_{\mathbf{Q}}$ on $\widehat{\mathbf{g}_{\mathbf{Q}}}$. We find that the following holds:

1. If $\tilde{\lambda} \in \widehat{\mathbf{g}_{\mathbf{Q}}}$, then the coadjoint orbit closure containing $\tilde{\lambda}$ is of the form $\tilde{\lambda}+\mathbf{i}_{\lambda}^{\perp}$, where $\mathbf{i}_{\lambda} \subseteq \mathbf{g}_{\mathbf{Q}}$ is the largest ideal contained in the subalgebra $\mathbf{r}_{\lambda}=\left\{X \in \mathbf{g}_{\mathbf{Q}} \mid \tilde{\lambda}([X, Y])=1\right.$, all $\left.Y \in \mathbf{g}_{\mathbf{Q}}\right\}$. We note that since $\mathbf{i}_{\lambda}$ is an ideal in $\mathbf{r}_{\lambda}$, the radical of the character $\tilde{\lambda}$, we have $\tilde{\lambda}\left(X^{*} Y\right)=\tilde{\lambda}(X+Y)$ for $X, Y \in \mathbf{i}_{\lambda}$; therefore we may regard $\lambda$, the restriction of $\tilde{\lambda}$ to $\mathbf{i}_{\lambda}$, as a character on the normal subgroup $I_{\lambda}$ of $G_{\mathbf{Q}}$. Let $K_{\lambda}$ be the kernel in $I_{\lambda}$ of $\lambda$; then $\left(G_{\mathbf{Q}}, I_{\lambda}\right) \subseteq K_{\lambda}$.

2. The representation $\tau_{\lambda}=\operatorname{Ind}\left(\lambda, I_{\lambda} \uparrow G_{\mathbf{Q}}\right)$ is a type II traceable factor representation which is canonically associated with the coadjoint orbit closure containing $\tilde{\lambda}$; there is a simple formula for the trace of $\tau_{\lambda}$ as an integral over the orbit closure.

3. A concrete Plancherel formula is proven for $G_{\mathbf{Q}}$; Plancherel measure is normalized Haar measure on $\widehat{Z\left(G_{\mathbf{Q}}\right)}$, the Pontryagin dual of the center of $\mathbf{g}_{\mathbf{Q}}$. (This is in contrast with other countable discrete groups, for example the free group on two generators, where Plancherel measure is atomic measure on one point; see [Mac].)

We construct the Pontryagin dual of $\mathbf{g}_{\mathbf{Q}}$ as follows. Let $\mathbf{A}$ denote the ring of adeles over the rationals (a standard reference for harmonic analysis on the ring of adeles is Tate's thesis, in [CF]); the rationals embed diagonally as a cocompact, discrete additive subgroup. Then $\widehat{\mathbf{Q}} \cong \mathbf{A} / \mathbf{Q}$, when $\mathbf{Q}$ is regarded as a discrete countable abelian group. If $\left\{X_{1}, \ldots, X_{n}\right\}$ is a basis for $\mathbf{g}_{\mathbf{Q}}$ over $\mathbf{Q}$, then we have the isomorphism

$$
\begin{aligned}
(\mathbf{A} / \mathbf{Q})^{n} & \rightarrow \widehat{\mathbf{g Q}}, \\
\left(\alpha_{1}, \ldots, \alpha_{n}\right) & \mapsto \tilde{\lambda}
\end{aligned}
$$

where $\tilde{\lambda}\left(q_{1} X_{1}+\cdots+q_{n} X_{n}\right)=\chi\left(\alpha_{1} q_{1}+\cdots+\alpha_{n} q_{n}\right)$ for some standard character $\chi \in \widehat{\mathbf{A}}$, usually taken to be the product of the standard characters on each component $\mathbf{Q}_{p}$ (i.e., those characters which map $\mathbf{Z}_{p}$ to 1$)$.

In proving the assertion about the nature of the orbit closures $\widehat{\mathbf{g}_{\mathbf{Q}}}$, we use the following result from [CP].

TheOREM. Let $\alpha_{1}, \ldots, \alpha_{n}$ be non-rational elements of $\mathbf{A}$. Then the set

$$
\left\{\left(\alpha_{1} x, \alpha_{2} x^{2}, \ldots, \alpha_{n} x^{n}\right), x \in \mathbf{Q}\right\}
$$

is dense in $(\mathbf{A} / \mathbf{Q})^{n}$. More generally, let $p_{1}(x), \ldots, p_{n}(x)$ be polynomials with coefficients in $\mathbf{A} / \mathbf{Q}$, having no constant terms, which are lin- 
early independent over $\mathbf{Q}$. Then the set $\left\{\left(p_{1}(x), \ldots, p_{n}(x)\right): x \in \mathbf{Q}\right\}$ is dense in $(\mathbf{A} / \mathbf{Q})^{n}$.

2. Traceable factor representations associated with coadjoint orbit closures. Recall that the representation $\tau_{\lambda}$ is a factor representation if $C R\left(\tau_{\lambda}\right)=\tau_{\lambda}\left(G_{\mathbf{Q}}\right)^{\prime} \cap \tau_{\lambda}\left(G_{\mathbf{Q}}\right)^{\prime \prime}=\mathbf{C I}\left(\mathscr{A}^{\prime}\right.$ denotes the commutator of the set $\mathscr{A})$.

LEMMA 1. Let $U \in \tau_{\lambda}\left(G_{\mathbf{Q}}\right)^{\prime}$, and let $H_{\lambda}$ be the Mackey space for the representation $\tau_{\lambda}$. Then $U$ is entirely determined by its value on the function $\delta_{1} \in H_{\lambda}$ defined by

$$
\delta_{1}(z)= \begin{cases}0, & z \notin I_{\lambda}, \\ \lambda(z), & z \in I_{\lambda} .\end{cases}
$$

Proof. We have that

$$
H_{\lambda}:=\left\{f: G_{\mathbf{Q}} \rightarrow \mathbf{C}\right\}
$$

where

$$
\begin{gathered}
\int_{G_{\mathbf{Q}} / I_{\lambda}}|f|^{2}<\infty, \\
f(k z)=\lambda(k) f(z), \quad k \in I_{\lambda}, z \in G_{\mathbf{Q}} .
\end{gathered}
$$

Let $H_{\lambda}^{\prime}=\left\{f \in H_{\lambda}: \int_{G_{\mathbf{Q}} \backslash I_{\lambda}}|f|<\infty\right\}$. Then $H_{\lambda}^{\prime}$ is a convolution algebra, with

$$
f * g(z)=\int_{G_{\mathbf{Q}} \backslash I_{\lambda}} f\left(z x^{-1}\right) g(x) d x
$$

well-defined and in $H_{\lambda}^{\prime}$. To see this, we must check that the integrand is constant on cosets of $I_{\lambda}$. Let $x, z \in G_{\mathbf{Q}}, t \in I_{\lambda}$. Then

$$
f\left(x(t z)^{-1}\right) g(t z)=f\left(x z^{-1} t^{-1}\right) g(t z)=f\left(t^{-1}\left(t x z^{-1} t^{-1}\right)\right) g(t z) .
$$

We have that $t \in I_{\lambda}$, so $t x z^{-1} t^{-1}=r x z^{-1}$, with $r=t x z^{-1} E^{1}\left(x z^{-1}\right)^{-1}$. Since $r$ is in the commutator of $G_{Q}$ with $I_{\lambda}, r \in K_{\lambda}$. Therefore

$$
\begin{aligned}
f\left(x z^{-1} t^{-1}\right) g(t z) & =f\left(t^{-1} r x z^{-1}\right) g(t z) \\
& =\lambda\left(t^{-1}\right) \lambda(r) \lambda(t) f\left(x^{-1} z\right) g(z)=f\left(x^{-1} z\right) g(z) .
\end{aligned}
$$

We note also that the convolution of an element in $H_{\lambda}$ with an element in $H_{\lambda}^{\prime}$ is again in $H_{\lambda}$. 

then

We have that $\delta_{1} * g=g$ for $g \in H_{\lambda}^{\prime}$. If $U$ commutes with $\tau_{\lambda}$,

$$
\begin{aligned}
U(g) & =U\left(\delta_{1} * g\right) \\
& =U\left(\int_{G_{\mathbf{Q}} \backslash I_{\lambda}} \delta_{1}\left(z x^{-1}\right) g(x) d x\right) \\
& =\int_{G_{\mathbf{Q}} \backslash I_{\lambda}} U\left(\delta_{1}\left(z x^{-1}\right)\right) g(x) d x \\
& =\int_{G_{\mathbf{Q}} \backslash I_{\lambda}} U\left(\tau_{\lambda}\left(x^{-1}\right) \delta_{1}(z)\right) g(x) d x \\
& =\int_{G_{\mathbf{Q}} \backslash I_{\lambda}} \tau_{\lambda}\left(x^{-1}\right) U\left(\delta_{1}(z)\right) g(x) d x \\
& =\int_{G_{\mathbf{Q}} \backslash I_{\lambda}} U\left(\delta_{1}\right)\left(z x^{-1}\right) g(x) d x=\left(U\left(\delta_{1}\right) * g\right)(z) .
\end{aligned}
$$

So $U$ restricted to $H_{\lambda}^{\prime}$ is really a convolution operator by the element $U\left(\delta_{1}\right) \in H_{\lambda}$. Since $H_{\lambda}^{\prime}$ is dense in $H_{\lambda}, U\left(\delta_{1}\right)$ determines $U$ on all of $H_{\lambda}$. This completes the proof of Lemma 1.

LEMMA 2. If $U \in C R\left(\tau_{\lambda}\right)$, then $U$ is convolution by an element of $H_{\lambda}$ which is constant on conjugacy classes. Furthermore, if $f \in H_{\lambda}$ is constant on conjugacy classes of $G_{\mathbf{Q}}$, and if convolution by $f$ is a bounded operator on $H_{\lambda}$, then convolution by $f$ is in $C R\left(\tau_{\lambda}\right)$.

Proof. We show the first part of Lemma 2 by evaluating $U\left(\delta_{1}\right)$ for $U \in C R\left(\tau_{\lambda}\right)$; if $\lambda_{x}, \rho_{x}$ are respectively the left and right regular representations of $G_{\mathbf{Q}}$ on $H_{\lambda}$, then both commute with $U \in C R\left(\tau_{\lambda}\right)$. Thus we have that

$$
U\left(\delta_{1}\right)(z)=U\left(\lambda_{x} \rho_{x} \delta_{1}\right)(z)=\lambda_{x} \rho_{x} U \delta_{1}(z)=U\left(\delta_{1}\left(x z x^{-1}\right)\right) .
$$

The second part of Lemma 2 follows when we note that $\tau_{\lambda}^{\prime}$ is the strong operator closure of left translation operators on $H_{\lambda}$. So $U \in \tau_{\lambda}^{\prime \prime}$ if and only if $\lambda(g) U=U \lambda(g)$ for all $g \in G_{\mathbf{Q}}$. Therefore, convolution by $f \in H_{\lambda}$ satisfying the boundedness condition is in $C R\left(\tau_{\lambda}\right)$ if and only if it commutes with both left and right translation, and we have seen that this holds if $f$ is constant on conjugacy classes. This completes Lemma 2.

LEMMA 3. Let $I_{\lambda}^{(2)}$ be the inverse image in $G_{\mathbf{Q}}$ of the center of $G_{\mathbf{Q}} \backslash I_{\lambda}$. Then $\tau_{\lambda}$ is a factor representation if and only if for all $\gamma \in$ 
$I_{\lambda}^{(2)}-I_{\lambda}$, the conjugacy class $C(\gamma) \subseteq G_{\mathbf{Q}}$ is not contained in the set $\gamma K_{\lambda}$.

Proof. Suppose $C(\gamma) \subseteq \gamma K_{\lambda}$. Then the function

$$
\delta_{\gamma}(x)= \begin{cases}\lambda(z) & \text { if } x=z \gamma, \quad z \in I_{\lambda}, \\ 0 & \text { otherwise }\end{cases}
$$

is constant on conjugacy classes and is an element of $H_{\lambda}^{\prime}$. Thus, by Lemma 2, it is in $C R\left(\tau_{\lambda}\right)$, but it is not a multiple of the identity, and so $\tau_{\lambda}$ is not a factor representation.

Conversely suppose $C(\gamma) \nsubseteq \gamma K_{\lambda}$, for all $\gamma \in I_{\lambda}^{(2)}-I_{\lambda}$. Then elements of $H_{\lambda}$ cannot be constant on $C(\gamma)$. If $x \in G_{\mathbf{Q}}-I_{\lambda}^{(2)}$, then $C(x)$ intersects infinitely many cosets of $I_{\lambda}$. To see this, note that $G_{\mathbf{Q}}$ acts on $G_{\mathbf{Q}} \backslash I_{\lambda}$ by inner automorphisms; consider the orbit of $x I_{\lambda}$. Since $x \notin I_{\lambda}^{(2)}, y x y^{-1} \notin x I_{\lambda}$ for some $y \in G_{\mathbf{Q}}$; therefore the isotropy group of $x$ under this action is a proper subgroup of $G_{\mathbf{Q}}$. Since $G_{\mathbf{Q}}$ contains no finite index subgroups, the orbit of $x I_{\lambda}$ is infinite, and so the conjugacy class of $x$ intersects infinitely many cosets of $I_{\lambda}$. Therefore, a function constant on $C(x)$ could not be in $H_{\lambda}$.

Thus, a function in $H_{\lambda}$ constant on conjugacy classes could be nonzero only on $I_{\lambda}$, and so would have to be a multiple of $\delta_{1} \in H_{\lambda}$. This completes the proof of Lemma 3.

The Pontryagin dual $\widehat{\mathbf{g}_{\mathbf{Q}}}$ of the discrete Lie algebra $\mathbf{g}_{\mathbf{Q}}$ is isomorphic to $(\mathbf{A} / \mathbf{Q})^{n}$, where $n$ is the dimension of $\mathbf{g}_{\mathbf{Q}}$ as a vector space. The coadjoint orbits of $G_{\mathbf{Q}}$ in $\widehat{\mathbf{g}_{\mathbf{Q}}}$ are given by polynomials $P_{i}, i=1, \ldots, n$, where $P_{i}: G_{\mathbf{Q}} \rightarrow \mathbf{A} / \mathbf{Q}$ are polynomials in $\mathbf{Q}^{n}$ with coefficients in $\mathbf{A} / \mathbf{Q}$. In what follows, we rely heavily upon the technical results in $[\mathbf{C P}]$.

THEOREM 1. Suppose $\tilde{\lambda} \in \widehat{\mathbf{g}_{\mathbf{Q}}}$. Then the closure of $\operatorname{Ad}^{*}\left(G_{\mathbf{Q}}\right) \tilde{\lambda}$ is "flat"; i.e., there exists a subgroup $V \cong \mathbf{A} / \mathbf{Q}^{k}$ of $\widehat{\mathbf{g}_{\mathbf{Q}}}$ such that the closure of the orbit is $\tilde{\lambda} V$.

Define $P\left(\mathbf{Q}^{k}, \mathbf{A} / \mathbf{Q}^{n}\right)$ to be the space of $n$-tuples of polynomials in $k$ rational variables, with coefficients in $\mathbf{A} / \mathbf{Q}$. We have that $\widehat{\mathbf{Q}}^{k} \cong$ $(\mathbf{A} / \mathbf{Q})^{k}$. Conversely, the Pontryagin dual of $(\mathbf{A} / \mathbf{Q})^{k}$ is $\mathbf{Q}^{k}$.

Lemma 4. Suppose $\vec{p}=\left(p_{1}(x), \ldots, p_{n}(x)\right) \in P\left(\mathbf{Q},(\mathbf{A} / \mathbf{Q})^{k}\right)$, where the $p_{i}$ have no constant terms. Then $\vec{p}(\mathbf{Q})$ is dense in some subgroup $V \subseteq(\mathbf{A} / \mathbf{Q})^{n}$, with $V \cong(\mathbf{A} / \mathbf{Q})^{k}$. 
Proof. First suppose that the $p_{i}$ are linearly independent over $\mathbf{Q}$. Then the closure of the image of $\mathbf{Q}$ under $\vec{p}$ is $(\mathbf{A} / \mathbf{Q})^{n}$, since the conclusion of Theorem 2 in [CP] holds as well if the polynomial vector $\left(p_{1}, \ldots, p_{n}\right)$ consists of polynomials which are linearly independent over $\mathbf{Q}$, so that no $\mathbf{Q}$-linear combination of the $p_{i}$ is 0 .

Now suppose that $\vec{p}=\left(p_{1}, \ldots, p_{n}\right)$ has Q-linearly dependent entries. Then the map

$$
\begin{aligned}
T_{\vec{p}}: \mathbf{Q}^{n} & \rightarrow P(\mathbf{Q}, \mathbf{A} / \mathbf{Q}), \\
\vec{q} & \mapsto \vec{q} \cdot \vec{p}
\end{aligned}
$$

has a nontrivial kernel $K$; it is a vector subspace of $\mathbf{Q}^{n}$. Then $\mathbf{Q}^{n} \backslash K$ is the dual of a subgroup $K^{\perp} \subseteq(\mathbf{A} / \mathbf{Q})^{n}$ having annihilator $K$; since $K$ is $\mathbf{Q}^{s}$ for some $s, K^{\perp} \cong(\mathbf{A} / \mathbf{Q})^{n-s}$. We see easily that $\vec{p}(\mathbf{Q}) \subseteq K^{\perp}$. Take a preimage for a basis of $\mathbf{Q}^{n} \backslash K$ in $\mathbf{Q}^{n},\left\{\vec{q}_{i}\right\}_{i=1}^{n-s}$, and define the map

$$
\begin{aligned}
\pi: K^{\perp} & \rightarrow(\mathbf{A} / \mathbf{Q})^{n-s}, \\
\vec{\alpha}=\left(\alpha_{1}, \ldots, \alpha_{n}\right) & \mapsto\left(\vec{q}_{1} \cdot \vec{\alpha}, \ldots, \vec{q}_{s} \cdot \vec{\alpha}\right) .
\end{aligned}
$$

Now consider $\pi \circ \vec{p}: \mathbf{Q} \rightarrow(\mathbf{A} / \mathbf{Q})^{n-s}$. The components of $\pi \circ \vec{p}$ form a linearly independent set of polynomials in $(\mathbf{A} / \mathbf{Q})^{n-s}$ without constant term, so $\pi \circ \vec{p}(\mathbf{Q})$ is dense in $(\mathbf{A} / \mathbf{Q})^{n-s}$. We can show that in fact $\pi$ is a homeomorphism of $K^{\perp}$ onto $(\mathbf{A} / \mathbf{Q})^{n-s}$. It is injective, since if $\vec{q}_{i} \cdot \vec{\alpha} \equiv \mathbf{Q}$ for all $i=1, \ldots, n-s$, we have that $\vec{q} \cdot \vec{\alpha} \equiv \mathbf{Q}$ for all $\vec{q} \in \mathbf{Q}^{n}$, so that $\vec{\alpha} \equiv 0$ in $(\mathbf{A} / \mathbf{Q})^{n}$. It is clearly a continuous map, and is onto; the image of $K^{\perp}$ contains a dense set in $(\mathbf{A} / \mathbf{Q})^{n-s}$ and is closed, since $K^{\perp}$ is compact. Thus, the image of $\mathbf{Q}$ under $\vec{p}$ is dense in $K^{\perp}$.

This completes the proof of Lemma 4; we now consider the case where the polynomials under consideration are of several rational variables.

Lemma 5. Let $\left\{p_{i}\right\}_{i=1}^{n} \subseteq P\left(\mathbf{Q}^{k}, \mathbf{A} / \mathbf{Q}\right)$ be a linearly independent set of polynomials in $k$ rational variables without constant terms; then the image of $\mathbf{Q}^{k}$ under $\vec{p}=\left(p_{1}, \ldots, p_{n}\right)$ is dense in $(\mathbf{A} / \mathbf{Q})^{n}$.

Proof. We will show that there is a map $\phi: \mathbf{Q} \rightarrow \mathbf{Q}^{k}, \phi(q)=$ $\left(q^{N_{1}}, \ldots, q^{N_{k}}\right)$, such that the $p_{i} \circ \phi$ form a linearly independent set in $P(\mathbf{Q}, \mathbf{A} / \mathbf{Q})$. The result follows immediately.

$\mathbf{N}$ will denote the natural numbers, including 0 . We define an order on $\mathbf{N}^{k}$ as follows: let $i \in 1, \ldots, k$ be the greatest integer satisfying $m_{i} \neq m_{i}^{\prime}$; then $\left(m_{1}, \ldots, m_{k}\right)>\left(m_{1}^{\prime}, \ldots, m_{k}^{\prime}\right)$ if $m_{i}>m_{i}^{\prime}$. 
We will define the degree of a monomial $x_{1}^{m_{1}} \cdots x_{k}^{m_{k}}$ to be $\left(m_{1}, \ldots, m_{k}\right)$, and the degree of a polynomial to be the degree of its highest-valued monomial. Then we may assume without loss of generality, by interchanging $p_{i}$ and $p_{j}$ and by adding rational multiples of $p_{i}$ to $p_{j}$, that the $\left\{p_{i}\right\}$ satisfy

1. $\operatorname{deg} p_{i} \geq \operatorname{deg} p_{j}$ if $i<j$

2. if $\operatorname{deg} p_{i}=\operatorname{deg} p_{i+1}=\cdots=\operatorname{deg} p_{j}$, then the leading coefficients (i.e., the coefficients of the monomials of highest degree in each polynomial) form a linearly independent set of adeles over $\mathbf{Q}$.

A simple induction argument shows the following:

3. For each finite set $\left\{M_{i}\right\} \in \mathbf{N}^{k}$, there exists an element $N=$ $\left(N_{1}, \ldots, N_{k}\right) \in \mathbf{N}^{k}$ such that $N \cdot M_{i}>N \cdot M_{j}$ if $M_{i}>M_{j}$ in the order on $\mathbf{N}^{k}(M \cdot N$ denotes the usual dot product).

Now let $M_{i}=\operatorname{deg} p_{i}$, and let $N$ be as in statement 3. For $x \in \mathbf{Q}$, let $\phi(x)=\left(x^{N_{1}}, \ldots, x^{N_{k}}\right)$. Then $\left\{p_{i} \circ \phi\right\}$ is a set of polynomials in one variable, with $\operatorname{deg} p_{i} \circ \phi>\operatorname{deg} p_{j} \circ \phi$ if and only if $\operatorname{deg} p_{i}>\operatorname{deg} p_{j}$; furthermore, the leading coefficients of the polynomials $p_{i} \circ \phi$ of a given degree form a linearly independent set over $\mathbf{Q}$ in $\mathbf{A} / \mathbf{Q}$. Thus the polynomials $p_{i} \circ \phi$ form a linearly independent set in $P(\mathbf{Q}, \mathbf{A} / \mathbf{Q})$.

LEMMA 6. Let $\vec{p}=\left(p_{1}, \ldots, p_{n}\right) \in P\left(\mathbf{Q}^{k},(\mathbf{A} / \mathbf{Q})^{n}\right)$, where the $p_{i}$ are polynomials without constant term. Then the closure in $(\mathbf{A} / \mathbf{Q})^{n}$ of the image of $\mathbf{Q}^{n}$ under this polynomial is a subgroup $V \cong(\mathbf{A} / \mathbf{Q})^{s}$ for some $s \leq k$. Furthermore, $V$ is the subgroup of $(\mathbf{A} / \mathbf{Q})^{k}$ annihilated by $K=\operatorname{ker} T_{\vec{p}}$ where

$$
\begin{aligned}
T_{\vec{p}}: \mathbf{Q}^{n} & \rightarrow P\left(\mathbf{Q}^{k}, \mathbf{A} / \mathbf{Q}\right), \\
\vec{q} & \mapsto \vec{q} \cdot \vec{p} .
\end{aligned}
$$

Proof. Lemma 5 gives this result for the case when $K$ is trivial. In general, we see easily that if $K=\operatorname{ker} T_{\vec{p}}$, then $\vec{p}\left(Q^{k}\right) \subseteq K^{\perp}$. Define $\pi: K^{\perp} \rightarrow(\mathbf{A} / \mathbf{Q})^{n-s}$ as in the proof of Lemma 4; then the components of $\pi \circ \vec{p}$ form a linearly independent set of polynomials in $(\mathbf{A} / \mathbf{Q})^{n-s}$. We now use Lemma 5 to show that the image of $\pi \circ \vec{p}\left(\mathbf{Q}^{k}\right)$ is dense in $(\mathbf{A} / \mathbf{Q})^{n-s}$, and proceed as in the proof of Lemma 4 to show that $\vec{p}\left(\mathbf{Q}^{k}\right)$ is dense in $K^{\perp}$. This completes the proof of Lemma 6 .

To complete the proof of Theorem 1, we take a strong Malcev basis $\left\{X_{1}, \ldots, X_{n}\right\}$ of $\mathbf{g}_{\mathbf{Q}}$ and write the elements of $\mathbf{g}_{\mathbf{Q}}$ with respect to this basis as $\left(x_{1}, \ldots, x_{n}\right)$. Recall that the base set of $G_{\mathbf{Q}}$ is $\mathbf{g}_{\mathbf{Q}}$ and that the exponential map with respect to our realization of $G_{\mathbf{Q}}$ is the 
identity on $\mathbf{g}_{\mathbf{Q}}$. Writing the elements of $\widehat{\mathbf{g}_{\mathbf{Q}}} \cong \mathbf{A} / \mathbf{Q}^{n}$ additively, we have that

$$
\begin{aligned}
\operatorname{Ad}^{*}\left(x_{1}, \ldots x_{n}\right) \tilde{\lambda} & =\tilde{\lambda}+\sum_{k=1}^{n} \frac{\operatorname{ad}^{*(k)}\left(x_{1}, \ldots, x_{n}\right)}{k !} \tilde{\lambda} \\
& =\tilde{\lambda}+\sum_{k=1}^{n} \vec{p}_{k}\left(x_{1}, \ldots, x_{n}\right)
\end{aligned}
$$

where the $\vec{p}_{k}$ are polynomial vectors in $P\left(\mathbf{Q}^{n}, \mathbf{A} / \mathbf{Q}^{n}\right)$ without constant terms. Lemma 6 now shows that the closure of the orbit $\operatorname{Ad}^{*}\left(G_{\mathbf{Q}}\right) \tilde{\lambda}$, again written additively, is $\tilde{\lambda}+V$ for some subgroup $V \cong \mathbf{A} / \mathbf{Q}^{s}$.

This completes the proof of Theorem 1 .

Let $I \subset \mathbf{g}_{\mathbf{Q}}$ be an ideal of $\mathbf{g}_{\mathbf{Q}}$. Let $I^{\perp}$ denote that subgroup of $\widehat{\mathbf{g}_{\mathbf{Q}}}$ which annihilates $I$. We will now prove

TheOREM 2. $\tau_{\lambda}=\operatorname{Ind}\left(I_{\lambda} \uparrow G_{\mathbf{Q}}, \lambda\right)$ is a factor representation.

We first prove the following lemma.

LEMMA 7. The closure in $\widehat{\mathbf{g Q}}$ of the coadjoint orbit of $\tilde{\lambda}$ is $\tilde{\lambda}+\mathbf{i} \frac{\perp}{\lambda}$.

Proof. From Theorem 1, we know that the closure of the orbit is a "flat", $\tilde{\lambda}+W_{0}$. Suppose that $W \in \mathbf{i}_{\lambda}$ and $Y \in \mathbf{g}_{\mathbf{Q}}$. Then

$$
\begin{aligned}
\operatorname{Ad}^{*}(\exp (-Y)) \tilde{\lambda}(W) & =\tilde{\lambda}(\operatorname{Ad}(\exp (Y) W)) \\
& =\tilde{\lambda}\left(W+[Y, W]+\frac{[Y,[Y, W]]}{2}+\cdots\right) .
\end{aligned}
$$

However, $[Y, W],[Y,[Y, W]]$, etc., are in $\left[\mathbf{i}_{\lambda}, \mathbf{g}_{\mathbf{Q}}\right]$, and so the above is just $\lambda(W)$. So $\operatorname{Ad}^{*}\left(G_{\mathbf{Q}}\right) \tilde{\lambda} \in \tilde{\lambda}+\mathbf{i}_{\lambda}^{\perp}$.

Conversely, consider the group $V_{0} \subseteq \widehat{\mathbf{g}_{\mathbf{Q}}}$ generated by the elements $\operatorname{Ad}^{*}\left(G_{\mathbf{Q}}\right) \tilde{\lambda}-\tilde{\lambda}$. We have $V_{0} \subseteq W_{0}$, since the coadjoint orbit closure is a flat. A typical element is

$$
\operatorname{ad}^{*}(Y) \tilde{\lambda}+\frac{\operatorname{ad}^{*(2)}(Y)}{2} \tilde{\lambda}+\cdots
$$

where the sum is finite. By taking linear combinations of these elements with $Y=X, 2 X, \ldots$ one can see that the individual terms are in $V_{0}$. So for each $Y \in \mathbf{g}_{\mathbf{Q}}, \operatorname{ad}^{*}(r Y) \lambda \in V_{0}$ for all $r \in \mathbf{Q}$. Note also that terms of the form $\operatorname{Ad}^{*}(x) \operatorname{Ad}^{*}(y) \lambda-\operatorname{Ad}^{*}(y) \lambda$ are in $V_{0}$, since $\operatorname{Ad}^{*}(x y) \lambda-\lambda$ and $\operatorname{Ad}^{*}(y) \lambda-\lambda$ are in $V_{0}$. 
The closure of $V_{0}$ is $V_{0}^{\perp \perp}$, so it suffices to show that $V_{0}^{\perp}=\mathbf{i}_{\lambda}$. We have seen that $\mathbf{i}_{\lambda} \subseteq V_{0}^{\perp}$. If $X \in V_{0}^{\perp}$, then $\operatorname{ad}^{*}(Y) \tilde{\lambda}(X)=1$ for all $Y \in \mathbf{g}_{\mathbf{Q}}$, so $\tilde{\lambda}([X, Y])=1$, and so $X \in \mathbf{r}_{\lambda}$. The same holds if we replace $\tilde{\lambda}$ with $\operatorname{Ad}^{*}(y) \tilde{\lambda}$, for $y \in G_{\mathbf{Q}}$, so $X \in \mathbf{r}_{\operatorname{Ad}^{*}(y) \tilde{\lambda}}=\operatorname{Ad}(y) \mathbf{r}_{\lambda}$. That is, $X \in \bigcap_{y \in G_{\mathbf{Q}}} \operatorname{Ad}(y) \mathbf{r}_{\lambda}=\mathbf{i}_{\lambda}$, the largest ideal of $\mathbf{g}_{\mathbf{Q}}$ contained in $\mathbf{r}_{\lambda}$. This completes the proof of Lemma 7 .

We now prove Theorem 2. Let $\gamma=\exp (\Gamma)$ be in $I_{\lambda}^{(2)}-I_{\lambda}$. Then since $\operatorname{Ad}^{*}\left(G_{\mathbf{Q}}\right) \tilde{\lambda}-\tilde{\lambda}=\mathbf{i}_{\lambda}^{\perp}$, the function

$$
x \mapsto\left(\operatorname{Ad}^{*}(x) \lambda-\lambda\right)(\Gamma)
$$

is not identically 1 as a function of $x \in G_{\mathbf{Q}}$. We may write this as

$$
\begin{aligned}
& \operatorname{Ad}^{*}(\exp (X)) \tilde{\lambda}-\tilde{\lambda}(\Gamma) \\
& \quad=\tilde{\lambda}([X, \Gamma]+[X,[X, \Gamma]]+\cdots+[X, \ldots[X, \Gamma], \ldots]) .
\end{aligned}
$$

However, since $[X, \Gamma] \in \mathbf{i}_{\lambda}^{(2)}$, all the brackets after the first are in the kernel of $\lambda$; therefore

$$
\operatorname{Ad}^{*}(x) \tilde{\lambda}(\Gamma)=\lambda([X, \Gamma]) .
$$

This shows that $\operatorname{ad}\left(\mathbf{g}_{\mathbf{Q}}\right) \Gamma \nsubseteq \mathbf{k}_{\lambda}$. Therefore the conjugacy class of $\gamma$ is not contained in $\gamma K_{\lambda}$. By Lemma 3, $\tau_{\lambda}$ must be a factor. This proves Theorem 2.

Note that for any element $\chi$ in the closure of $\operatorname{Ad}^{*}\left(G_{\mathbf{Q}}\right) \tilde{\lambda}$, the restriction of $\chi$ to $\mathbf{i}_{\lambda}$ is $\lambda$. Thus we see that the factor representation $\tau_{\lambda}$ is canonically associated with the closure of the orbit $\operatorname{Ad}^{*}\left(G_{\mathbf{Q}}\right) \tilde{\lambda}$ for each $\tilde{\lambda} \in \widehat{\mathbf{g}_{\mathbf{Q}}}$.

3. The trace on the factor $\tau_{\lambda}$ and the Plancherel formula. In this section, we demonstrate that the factor representation $\tau_{\lambda}$ of the previous section is traceable, and develop an orbital trace formula and Plancherel formula.

Let $\tilde{\lambda} \in \widehat{\mathbf{g}_{\mathbf{Q}}}$; there is then an ideal $\mathbf{i}_{\lambda}$ of $\mathbf{g}_{\mathbf{Q}}$ such that the closure in $(\mathbf{A} / \mathbf{Q})^{n}$ of $\operatorname{Ad}^{*}\left(G_{\mathbf{Q}}\right) \tilde{\lambda}$ is $\tilde{\lambda}+\mathbf{i}_{\lambda}^{\perp}$. Note that on $\mathbf{i}_{\lambda}, \operatorname{Ad}^{*}(x) \tilde{\lambda}=\tilde{\lambda}$ for all $x \in G_{\mathbf{Q}}$.

Let $H_{\lambda}$ be the Mackey space for $\tau_{\lambda}$ as defined in the previous section. We define $\bar{G}_{\mathbf{Q}}=G_{\mathbf{Q}} \backslash I_{\lambda}$. Let $\alpha: \bar{G}_{\mathbf{Q}} \rightarrow G_{\mathbf{Q}}$ give a cross section of the cosets of $\bar{G}_{\mathbf{Q}}$ satisfying $\alpha(\bar{e})=e$ (where $e$ denotes the identity element of $G_{\mathbf{Q}}$ ) and $\alpha\left(\bar{x}^{-1}\right)=\alpha(\bar{x})^{-1}$. Then a basis for $H_{\lambda}$ is given by the functions $\delta_{\alpha(\bar{x})}$, where

$$
\delta_{\alpha(\bar{x})}(y)= \begin{cases}\tilde{\lambda}(z), & \text { if } y=\alpha(\bar{x}) z, \quad z \in I_{\lambda}, \\ 0, & \text { if } y \notin \alpha(\bar{x}) .\end{cases}
$$


Let $J_{\bar{x}}: \mathbf{C} \rightarrow H_{\lambda}$ map 1 to $\delta_{\alpha(\bar{x})}$. Then $J_{\bar{x}}^{*}(f)=f(\alpha(\bar{x}))$ for $f \in H_{\lambda}$. So if $A \in L\left(H_{\lambda}\right)$, the space of bounded linear operators on $H_{\lambda}$, and we write $A_{\bar{x}, \bar{y}}=J_{\bar{x}}^{*} A J_{\bar{y}}$, then we have

$$
\begin{aligned}
A \delta_{\alpha(\bar{y})}(\alpha(\bar{x})) & =\left(A \delta_{\alpha(\bar{y})}, \delta_{\alpha(\bar{x})}\right)=\left(A J_{\bar{y}}(1), J_{\bar{x}}(1)\right) \\
& =\left(J_{\bar{x}}^{*} A J_{\bar{y}}(1), 1\right)=A_{\bar{x}, \bar{y}} \in \mathbf{C} .
\end{aligned}
$$

We define $c: \bar{G}_{\mathbf{Q}} \times G_{\mathbf{Q}} \rightarrow I_{\lambda}$ by

$$
\alpha(\bar{x}) y=c(\bar{x}, y) \alpha(\overline{x y}) .
$$

We have the cocycle identity

$$
c(\bar{x}, u) c(\overline{x u}, v)=c(\bar{x}, u v) .
$$

Note that if $u \in I_{\lambda}$, then $\bar{x}=\overline{x u}$; therefore $c(\bar{x}, u)=\alpha(\bar{x}) u \alpha(\bar{x})^{-1}$, and we have

$$
\alpha(\bar{x}) u \alpha(\bar{x})^{-1} c(\bar{x}, v)=c(\bar{x}, u v)
$$

for $u \in I_{\lambda}$. We define $\xi(\bar{x}, y)=\lambda(c(\bar{x}, y))$; we then have, for $u \in I_{\lambda}$,

$$
\xi(\bar{x}, u)=\lambda\left(\alpha(\bar{x}) u \alpha\left(\bar{x}^{-1}\right)\right)=\operatorname{Ad}^{*}(\alpha(\bar{x}))(u)=\lambda(u),
$$

since $\operatorname{Ad}^{*}\left(G_{\mathbf{Q}}\right) \lambda \subseteq \lambda+\mathbf{i}_{\lambda}^{\perp}$. It follows that

$$
\xi(\bar{x}, v) \lambda(u)=\xi(\bar{x}, u v)=\xi(\bar{x}, v u)
$$

if $u \in I_{\lambda}$.

We now compute $\tau_{\lambda}(g)_{\bar{x}, \bar{y}}$ for $g \in G_{\mathbf{Q}}$. Since $\tau_{\lambda}(u g)=\lambda(u) \tau_{\lambda}(g)$ if $u \in I_{\lambda}$, it suffices to consider the case where $g=\alpha(\bar{g})$. We then have

$$
\tau_{\lambda}(g)_{\bar{x}, \bar{y}}=\tau_{\lambda}(g) \delta_{\alpha(\bar{y})}(\alpha(\bar{x}))=\delta_{\alpha(\bar{y})}(\alpha(\overline{x g}))= \begin{cases}0 & \text { if } \bar{y} \neq \overline{x g} \\ \xi(\bar{x}, g) & \text { if } \bar{y}=\overline{x g}\end{cases}
$$

because if $\bar{y}=\overline{x g}$, then $\alpha(\bar{x}) g=c(\bar{x}, g) \alpha(\overline{x g})=c(\bar{x}, g) \alpha(\bar{y})$, and $\delta_{\alpha(\bar{y})} c(\bar{x}, g) \alpha(y)=\lambda(c(\bar{x}, g))=\xi(\bar{x}, g)$.

Since we have chosen $g=\alpha(\bar{g})$, it follows that

$$
\tau_{\lambda}(g)_{\bar{x}, \bar{y}}= \begin{cases}\xi\left(\bar{x}, \alpha\left(\overline{x^{-1} y}\right)\right) & \text { if } \bar{g}=\overline{x^{-1} y} \\ 0 & \text { otherwise. }\end{cases}
$$

This shows that $\tau_{\lambda}(g)_{\bar{e}, \bar{g}}=\xi(\bar{e}, \alpha(\bar{g}))$. However, $\alpha(\bar{e}) \alpha(\bar{g})=\alpha(\bar{g})$, so that $c(\bar{e}, \alpha(\bar{g}))=e$ and $\xi(\bar{e}, \alpha(\bar{g}))=1$. We thus have

$$
\tau_{\lambda}(g)_{\bar{x}, \bar{y}}=\xi\left(\bar{x}, \alpha\left(\overline{x^{-1} y}\right)\right) \tau_{\lambda}(g)_{\bar{e}, \overline{x^{-1} y}}
$$

if $g=\alpha(\bar{g})$. 
This also holds, however, if $g=u \alpha(\bar{g}), u \in I_{\lambda}$; both sides are multiplied by a factor of $\lambda(u)$. It holds also if $\overline{x^{-1} y} \neq \bar{g}$, since then both sides are 0 . Therefore, (1) holds for all $\bar{x}, \bar{y} \in \bar{G}_{\mathbf{Q}}$. Since (1) depends linearly upon $\tau_{\lambda}(g)$, we have also

$$
\tau_{\lambda}(f)_{\bar{x}, \bar{y}}=\xi\left(\bar{x}, \alpha\left(\overline{x^{-1} y}\right)\right) \tau_{\lambda}(f)_{\bar{e}, \overline{x^{-1} y}}
$$

for all $f \in C_{c}\left(G_{\mathbf{Q}}\right)$. This relation is preserved under the taking of weak limits in $\tau_{\lambda}(f)$, so that (2) holds if $\tau_{\lambda}(f)$ is replaced by an element of the Von Neumann algebra generated by the $\tau_{\lambda}(f)$.

We now follow Dixmier ([Dx], I.4.2 and I.9.2). Let $\mathscr{A}$ be the Von Neumann algebra generated by the $\tau_{\lambda}(f)$, for $f \in C_{c}\left(G_{\mathbf{Q}}\right)$. We define $\phi: \mathscr{A} \rightarrow \mathbf{C}$ by $\phi(A)=A_{\bar{e}, \bar{e}}$. Then the following hold:

1. $\phi$ is linear.

2. $\phi\left(A^{*} A\right)=\phi\left(A A^{*}\right) \geq 0$, with equality only if $A=0$.

Proof of Claim 2. We have $A \delta_{\alpha(\bar{e})}=\sum_{\bar{x} \in \bar{G}_{\mathbf{Q}}} A_{\bar{x}, \bar{e}} \delta_{\alpha(\bar{x})}$, so that

$$
\left(A^{*} A\right)_{\bar{e}, \bar{e}}=\left(A \delta_{\alpha(\bar{e})}, A \delta_{\alpha(\bar{e})}\right)=\sum_{\bar{x} \in \bar{G}_{\mathbf{Q}}}\left|A_{\bar{x}, \bar{e}}\right|^{2} .
$$

Similarly,

$$
\left(A A^{*}\right)_{\bar{e}, \bar{e}}=\sum_{\bar{x} \in \bar{G}_{\mathbf{Q}}}\left|A_{\bar{x}, \bar{e}}^{*}\right|^{2} .
$$

To see that these sums are equal, we note that $A_{\bar{x}, \bar{y}}^{*}=\bar{A}_{\bar{y}}, \bar{x}$, so that in particular $A_{\bar{x}, \bar{e}}^{*}=\bar{A}_{\bar{e}, \bar{x}}$; also, from (2), $A_{\bar{x}, \bar{e}}=\xi\left(\bar{x}, \alpha\left(\bar{x}^{-1}\right)\right) A_{\bar{e}, \bar{x}^{-1}}$. Therefore, $\left|A_{\bar{x}, \bar{e}}^{*}\right|=\left|A_{\bar{e}}, \bar{x}\right|$ and $\left|A_{\bar{x}, \bar{e}}\right|=\left|A_{\bar{e}, \bar{x}^{-1}}\right|$. Thus the sums over $\bar{G}_{\mathbf{Q}}$ which determine $\phi\left(A^{*} A\right)$ and $\phi\left(A A^{*}\right)$ are the same.

For the second assertion, suppose $\phi\left(A A^{*}\right)=0$. Then $A_{\bar{x}, \bar{e}}=0$ for all $\bar{x} \in \bar{G}_{\mathbf{Q}}$. By (2), we see that then $A_{\bar{x}, \bar{y}}=0$ for all $\bar{x}, \bar{y} \in \bar{G}_{\mathbf{Q}}$. Hence $A=0$.

3. $\phi$ is normal, since $\phi(A)=\left(A \delta_{\alpha(\bar{e})}, \delta_{\alpha(\bar{e})}\right)$; see ([Dx], Theorem 1, I.4.2). Therefore, $\phi$ is (up to positive multiples) the unique trace on the factor $\mathscr{A}$.

To compute $\phi$ on $L^{1}\left(G_{\mathbf{Q}}\right)$, we compute it on $C_{c}\left(G_{\mathbf{Q}}\right)$ and then use the $L^{1}$ norm-continuity of $\phi$ to extend to $L^{1}\left(G_{\mathbf{Q}}\right)$. Since $\tau_{\lambda}\left(C_{c}\left(G_{\mathbf{Q}}\right)\right)$ consists of finite linear combinations of elements of $\tau_{\lambda}\left(G_{\mathbf{Q}}\right)$,we compute $\phi$ on $\tau_{\lambda}\left(G_{\mathbf{Q}}\right)$. We see that if $g \notin I_{\lambda}$, then $\phi\left(\tau_{\lambda}(g)\right)=0$, and if $g \in I_{\lambda}$, then $\phi\left(\tau_{\lambda}(g)\right)=\lambda(g)$. Therefore we have

$$
\phi(f)=\sum_{u \in I_{\lambda}} \lambda(u) f(u) .
$$


By linearity and continuity, (3) holds also for $f \in L^{1}\left(G_{\mathbf{Q}}\right)$.

Now suppose that $F \in L^{1}\left(\mathbf{g}_{\mathbf{Q}}\right)$, and that $f=F \circ \log \in L^{1}\left(G_{\mathbf{Q}}\right)$ (recall that, as we have defined it, log on the set level is just the identity). Let $\widetilde{F}(X)=\tilde{\lambda}(X) F(X)$. Then for $\chi \in \mathbf{i}_{\lambda}^{\perp}$,

$$
\widetilde{F}^{\wedge}(\chi)=\sum_{X \in \mathbf{g}_{\mathbf{Q}}} \widetilde{F}(X) \chi(X)=\sum_{X \in \mathbf{g}_{\mathbf{Q}}} F(X)(\chi+\tilde{\lambda})(X)=F^{\wedge}(\chi+\tilde{\lambda}) .
$$

Therefore,

$$
\begin{aligned}
\phi(f) & =\sum_{u \in I_{\lambda}} \lambda(u) f(u)=\sum_{Y \in \mathbf{i}_{\lambda}} \lambda(Y) F(Y)=\sum_{Y \in \mathbf{i}_{\lambda}} \widetilde{F}(Y) \\
& =\int_{\mathbf{i}_{\lambda}^{\perp}} \widetilde{F}^{\wedge}(\chi) d \chi=\int_{\mathbf{i}_{\lambda}^{\perp}} F^{\wedge}(\chi+\tilde{\lambda}) d \chi=\int_{\overline{\mathscr{O}}_{\lambda}} F^{\wedge}(\chi) d \chi .
\end{aligned}
$$

The last set of equations follows from Poisson summation on $\mathbf{i}_{\lambda}$. We note that the measure on the closure of $\mathscr{O}_{\lambda}$ is the lift of normalized Haar measure on $\mathbf{i}_{\lambda}^{\perp}$. This gives the orbital trace formula for $\tau_{\lambda}$.

THEOREM 3. Let $\phi$ be the unique trace on the factor $\mathscr{A}$. Then for $f \in L^{1}\left(G_{\mathbf{Q}}\right), \phi(f)$ is given by the orbital trace formula

$$
\phi(f)=\int_{\overline{\mathscr{O}}_{\lambda}} F^{\wedge}(\chi) d \chi,
$$

where $F=f \circ \exp \in L^{1}\left(\mathbf{g}_{\mathbf{Q}}\right)$.

We finish by developing a Plancherel formula for $G_{\mathbf{Q}}$. For $\tilde{\lambda} \in \widehat{\mathbf{g}_{\mathbf{Q}}}$, we call the closure of the $\operatorname{Ad}^{*}\left(G_{\mathbf{Q}}\right)$-orbit $\overline{\mathscr{O}}_{\lambda}$ of $\tilde{\lambda}$ generic if $\overline{\mathscr{O}}_{\lambda}=$ $\tilde{\lambda}+\mathbf{z}\left(\mathbf{g}_{\mathbf{Q}}\right)^{\perp}$, or equivalently if $\mathbf{i}_{\lambda}=\mathbf{z}\left(\mathbf{g}_{\mathbf{Q}}\right)$. We will refer to $\tilde{\lambda}$ as generic if the closure of its $G_{\mathbf{Q}}$-orbit is generic.

We define the rising central series of ideals of $\mathbf{g}_{\mathbf{Q}}$ as follows: let $\mathbf{g}_{0}=(\mathbf{0})$, and let $\pi_{0}$ be the identity map on $\mathbf{g}_{\mathbf{Q}}$. We then let $\pi_{k}: \mathbf{g}_{\mathbf{Q}} \rightarrow$ $\mathbf{g}_{\mathbf{Q}} \backslash \mathbf{g}_{k-1}$ be the natural map, and let $\mathbf{g}_{k}$ be the inverse image in $\mathbf{g}_{\mathbf{Q}}$ of the center of $\mathbf{g}_{\mathbf{Q}} \backslash \mathbf{g}_{k-1}$. Then since $\mathbf{g}_{\mathbf{Q}}$ is nilpotent, the rising central series terminates at some $\mathbf{g}_{n}=\mathbf{g}_{\mathbf{Q}}$.

Lemma 8. Suppose $\tilde{\lambda} \in \widehat{\mathbf{g Q}}$. Then $\tilde{\lambda}$ is generic if and only if there does not exist $X \in \mathbf{g}_{2}$ such that $\operatorname{ad}(X) \mathbf{g}_{\mathbf{Q}} \subseteq \operatorname{ker}(\tilde{\lambda})$.

Proof. If $\mathbf{i}_{\lambda}$ properly contains $\mathbf{z}\left(\mathbf{g}_{\mathbf{Q}}\right)$, then take $X \in \mathbf{i}_{\lambda} \cap \mathbf{g}_{2}$ such that $X \notin \mathbf{z}\left(\mathbf{g}_{\mathbf{Q}}\right)$. Then $\tilde{\lambda}\left(\left[X, \mathbf{g}_{\mathbf{Q}}\right]\right)=1$ by definition of $\mathbf{i}_{\lambda}$, so $\operatorname{ad}(X) \mathbf{g}_{\mathbf{Q}} \subseteq$ $\operatorname{ker}(\tilde{\lambda})$. Conversely, suppose there is an $X \in \mathbf{g}_{2}$ such that $\operatorname{ad}(X) \mathbf{g}_{\mathbf{Q}} \subseteq$ 
$\operatorname{ker}(\tilde{\lambda})$. Then $\mathbf{Q} X \oplus \mathbf{z}\left(\mathbf{g}_{\mathbf{Q}}\right) \subseteq \mathbf{r}_{\lambda}$ is an ideal of $\mathbf{g}_{\mathbf{Q}}$; hence it is in $\mathbf{i}_{\lambda}$, and $\mathbf{i}_{\lambda}$ properly contains $\mathbf{z}\left(\mathbf{g}_{\mathbf{Q}}\right)$. Thus $\tilde{\lambda}$ is not generic.

Note that the second condition of Lemma 7 implies that whether $\tilde{\lambda}$ is generic depends upon its restriction to $\mathbf{z}\left(\mathbf{g}_{\mathbf{Q}}\right)$; thus we will (by abuse of notation) refer to $\lambda \in \hat{\mathbf{z}}\left(\mathbf{g}_{\mathbf{Q}}\right)$ as generic if any (equivalently all) of its extensions to $g_{Q}$ are generic. We will show that for a set of $\lambda \in \hat{\mathbf{z}}\left(\mathbf{g}_{\mathbf{Q}}\right)$ of full Haar measure, the second condition of Lemma 7 holds; in fact, we will show something stronger than that.

LEMMA 9. The $\lambda \in \widehat{\mathbf{z}\left(\mathbf{g Q}_{\mathbf{Q}}\right)}$ with $\operatorname{ker} \lambda=0$ are of full Haar measure in $\widehat{\mathbf{z}\left(\mathbf{g}_{\mathbf{Q}}\right)}$.

Proof. Suppose $\mathbf{z}\left(\mathbf{g}_{\mathbf{Q}}\right) \cong \mathbf{Q}^{k}$, and let $\left\{Z_{1}, \ldots, Z_{k}\right\}$ be a basis over $\mathbf{Q}$ for $\mathbf{z}\left(\mathbf{g}_{\mathbf{Q}}\right)$. If $\lambda \in \mathbf{z}\left(\widehat{\left.\mathbf{g}_{\mathbf{Q}}\right)}\right.$, then we may identify $\lambda$ with an element $\vec{\alpha} \in(\mathbf{A} / \mathbf{Q})^{k}$ by

$$
\lambda\left(q_{1} Z_{1}+\cdots+q_{k} Z_{k}\right)=\chi\left(\alpha_{1} q_{1}+\cdots+\alpha_{k} q_{k}\right),
$$

where $\vec{\alpha}=\left(\alpha_{1}, \ldots, \alpha_{k}\right)$ and $\chi$ is the standard character on $\mathbf{A} / \mathbf{Q}$. This gives an isomorphism of $\mathbf{z}\left(\widehat{\left.\mathbf{g}_{\mathbf{Q}}\right)}\right.$ with $(\mathbf{A} / \mathbf{Q})^{k}$. We define, for $\vec{\alpha} \in(\mathbf{A} / \mathbf{Q})^{k}$ and $\vec{q} \in \mathbf{Q}^{k}$,

$$
\vec{\alpha} \cdot \vec{q}=\alpha_{1} q_{1}+\cdots+\alpha_{k} q_{k} \in \mathbf{A} / \mathbf{Q} \text {. }
$$

For an element $\vec{q} \in Q^{k}$, we define the linear map

$$
\begin{aligned}
T_{\vec{q}}:(\mathbf{A} / \mathbf{Q})^{k} & \rightarrow \mathbf{A} / \mathbf{Q}, \\
\vec{\alpha} & \mapsto \vec{\alpha} \cdot \vec{q} .
\end{aligned}
$$

The set of $\lambda$ with nontrivial kernel corresponds to the union of the kernels of $T_{\vec{q}}, \vec{q} \neq 0$. Since $\mathbf{Q}^{k}$ is countable, we need only show that each kernel is of Haar measure 0 in $(\mathbf{A} / \mathbf{Q})^{k}$.

Suppose now that $\vec{\alpha} \in \operatorname{ker} T_{\vec{q}}$. Then we have $\vec{\alpha} \cdot \vec{q} \equiv \mathbf{Q}$ in $\mathbf{A} / \mathbf{Q}$, and therefore we may take representatives $\alpha_{i}$ for each coordinate of $\vec{\alpha}$ in the adeles, so that

$$
\alpha_{1} q_{1}+\cdots+\alpha_{k} q_{k}=0 .
$$

This means that in the $\mathbf{Q}_{p}$ th coordinate,

$$
\left(\alpha_{1} q_{1}+\cdots+\alpha_{k} q_{k}\right)_{p}=0 .
$$

This condition is satisfied by elements $\vec{\alpha} \in(\mathbf{A} / \mathbf{Q})^{k}$ such that each $(\vec{\alpha})_{p}=\left(\left(\alpha_{1}\right)_{p}, \ldots,\left(\alpha_{k}\right)_{p}\right)$ lies in the hyperplane

$$
x_{1} q_{1}+\cdots+x_{k} q_{k}=0 \text {, }
$$


where $\left(x_{1}, \ldots, x_{k}\right) \in\left(\mathbf{Q}_{p}\right)^{k}$. Therefore, if $\vec{q} \neq \overrightarrow{0}$, the set of choices in each $\mathbf{Q}_{p}^{n}$ has Haar measure zero, and so the preimage of $\operatorname{ker} T_{\vec{q}}$ in the adeles has measure zero. Therefore the Haar measure of $\operatorname{ker} T_{\vec{q}}$ is zero. This completes the proof of Lemma 8 .

Now the Plancherel formula is nearly trivial. If $F \in C_{c}\left(\mathbf{g}_{\mathbf{Q}}\right)$, then $f=F \circ \log \in C_{c}\left(G_{\mathbf{Q}}\right)$. Let $\left\{Z_{1}, \ldots, Z_{k}, X_{k+1}, \ldots, X_{n}\right\}$ be an extension of the basis given above to a basis of $\mathbf{g}_{\mathbf{Q}}$. We then have an inclusion $\mathbf{z} \widehat{\mathbf{( \mathbf { g } _ { \mathbf { Q } } )}} \rightarrow \widehat{\mathbf{g}_{\mathbf{Q}}}$ as follows; $\lambda \mapsto \tilde{\lambda}$ if

$\tilde{\lambda}\left(q_{1} Z_{1}+\cdots+q_{k} Z_{k}+q_{k+1} X_{k+1}+\cdots+q_{n} Z_{n}\right)=\lambda\left(q_{1} Z_{1}+\cdots+q_{k} Z_{k}\right)$ for all $\vec{q} \in \mathbf{Q}^{k}$. Then

$$
f(0)=F(0)=\int_{\widehat{\mathbf{g}_{\mathbf{Q}}}} F^{\wedge}(\xi) d \xi=\int_{\widehat{\mathbf{z}\left(\mathbf{g}_{\mathbf{Q}}\right)}} \int_{\mathbf{z}\left(\mathbf{g}_{\mathbf{Q}}\right)^{\perp}} F^{\wedge}(\lambda+\chi) d \chi d \lambda,
$$

where all Haar measures are normalized so that their (compact) supports have measure 1 . We let $d_{\lambda}(\chi)$ be the lift of normalized Haar measure on $\mathbf{z}\left(\mathbf{g}_{\mathbf{Q}}\right)^{\perp}$ to $\lambda+\mathbf{z}\left(\mathbf{g}_{\mathbf{Q}}\right)^{\perp}$; if $\lambda$ is generic, this is $\overline{\mathscr{O}}_{\lambda}$. For a.e. $\lambda \in \mathbf{z}\left(\widehat{\mathbf{g}_{\mathbf{Q}}}\right), d_{\lambda}(\chi)$ is the orbital measure on $\overline{\mathscr{O}}_{\lambda}$ in the trace formula, so the above integral becomes

$$
\begin{aligned}
\int_{\widehat{\mathbf{z}\left(\mathbf{g}_{\mathbf{Q}}\right)}} & \left\{\int_{\mathbf{z}\left(\mathbf{g}_{\mathbf{Q}}\right)^{\perp}} F^{\wedge}(\lambda+\chi) d \chi\right\} d \lambda \\
= & \int_{\widehat{\mathbf{z}\left(\mathbf{g}_{\mathbf{Q}}\right)}}\left\{\int_{\overline{\mathscr{O}}_{\lambda}} F^{\wedge}(\chi) d_{\lambda}(\chi)\right\} d \lambda=\int_{\widehat{\mathbf{z}\left(\mathbf{g}_{\mathbf{Q}}\right)}} \phi_{\lambda}(f) d \lambda .
\end{aligned}
$$

Thus we see that Plancherel measure for $G_{\mathbf{Q}}$ is normalized Haar measure on $\widehat{\mathbf{z}\left(\mathbf{g}_{\mathbf{Q}}\right)}$. We have proved

Theorem 4 (Plancherel Formula). Suppose $f \in C_{c}\left(\mathbf{g}_{\mathbf{Q}}\right)$, and that for generic $\lambda \in \mathbf{z}\left(\widehat{\mathbf{g}_{\mathbf{Q}}}\right), \phi_{\lambda}$ is the trace associated with the factor representation $\tau_{\lambda}$ induced from the character $\lambda \in \mathbf{z}\left(\widehat{\left.\mathbf{g Q}_{\mathbf{Q}}\right)}\right.$. Then $\lambda \mapsto \phi_{\lambda}(f)$ is defined for a.a. $\lambda$, and is integrable on $\mathbf{z}\left(\widehat{\mathbf{g}_{\mathbf{Q}}}\right)$, and we have

$$
f(0)=\int_{\widehat{\mathbf{z}\left(\mathbf{g}_{\mathbf{Q}}\right)}} \phi_{\lambda}(f) d \mu(\lambda)
$$

where $\mu$ is Haar measure on $\mathbf{z}\left(\widehat{\mathbf{g}_{\mathbf{Q}}}\right)$, normalized so that $\mu\left(\mathbf{z}\left(\mathbf{g}_{\mathbf{Q}}\right)\right)=1$.

Editor's note. The mathematical community was deeply saddened by the death of Larry Corwin on March 19, 1992. Larry had made 
fundamental contributions to the harmonic analysis of $p$-adic reductive groups, nilpotent Lie groups and, as this paper illustrates, discrete groups. His friends also recall him as an effective administrator and innovative teacher, and remember his incredible wit, his playful and spontaneous sense of humor, and his remarkable energy and zest for life.

\section{REFERENCES}

[CF] J. Cassels and A. Frohlich, Algebraic Number Theory, Academic Press, London, 1967.

[CP] L. Corwin and C. Pfeffer, On the density of sets in $(\mathbf{A} / \mathbf{Q})^{n}$ defined by polynomials, to appear, Colloq. Math.

[Dx] J. Dixmier, Von Neumann Algebras, North-Holland Publishing Co., N.Y., 1969.

[Gdt] A. Guichardet, Caracteres des algebres de Banach involutives, Ann. Inst. Fourier (Grenoble), 13 (1963), 1-81.

[How1] R. Howe, On Frobenius reciprocity for unipotent algebraic groups over $\mathbf{Q}$, Amer. J. Math., 93 (1971), 163-172.

[How2] _-, On representations of discrete, finitely generated, torsion-free, nilpotent groups, Pacific J. Math., 73 (1977), 281-305.

[How3] _ The Fourier transform for nilpotent locally compact groups, I, Pacific J. Math., 73 (1977), 307-327.

[Mac] G. Mackey, The Theory of Group Representations, mimeographed notes, University of Chicago, 1955.

[Puk] L. Pukansky, Characters of connected Lie groups, Bull. Amer. Math. Soc., 80, No. 4 (1974), 709-712.

[Tho] E. Thoma, Über unitare Darstellungen abzahlbarer diskreter Gruppen, Math. Ann., 153 (1964), 111-141.

Received February 18, 1992.

Florida AtLantic University

BOCA RATON, FL 33431-0991 



\title{
PACIFIC JOURNAL OF MATHEMATICS
}

\author{
Founded by
}

\author{
E. F. BECKENBACH (1906-1982) F. Wolf (1904-1989)
}

\section{EDITORS}

Sun-Yung A. Chang

(Managing Editor)

University of California

Los Angeles, CA 90024-1555

chang@math.ucla.edu

\section{F. Michael Christ}

University of California

Los Angeles, CA 90024-1555

christ@math.ucla.edu

Herbert Clemens

University of Utah

Salt Lake City, UT 84112

clemens@math.utah.edu
THOMAS ENRIGHT

University of California, San Diego

La Jolla, CA 92093

tenright@ucsd.edu

Nicholas ERCOLANI

University of Arizona

Tucson, AZ 85721

ercolani@math.arizona.edu

R. FINN

Stanford University

Stanford, CA 94305

finn@gauss.stanford.edu

VAUGHAN F. R. JONES

University of California

Berkeley, CA 94720

vfr@math.berkeley.edu
SteVen KerCKHofF

Stanford University

Stanford, CA 94305

spk@gauss.stanford.edu

MARTIN ScharLemanN

University of California

Santa Barbara, CA 93106

mgscharl@math.ucsb.edu

Harold STARK

University of California, San Diego

La Jolla, CA 92093

V. S. VARADARAJAN

University of California

Los Angeles, CA 90024-1555

vsv@math.ucla.edu

\section{SUPPORTING INSTITUTIONS}

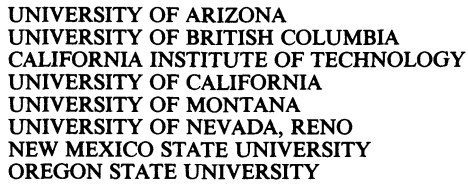

The Supporting Institutions listed above contribute to the cost of publication of this Journal, but they are not owners or publishers and have no responsibility for its content or policies.

Mathematical papers intended for publication in the Pacific Journal of Mathematics should be in typed form or offset-reproduced (not dittoed), double spaced with large margins. Please do not use built up fractions in the text of the manuscript. However, you may use them in the displayed equations. Underline Greek letters in red, German in green, and script in blue. The first paragraph must be capable of being used separately as a synopsis of the entire paper. In particular it should contain no bibliographic references. Please propose a heading for the odd numbered pages of less than 35 characters. Manuscripts, in triplicate, may be sent to any one of the editors. Please classify according to the 1991 Mathematics Subject Classification scheme which can be found in the December index volumes of Mathematical Reviews. Supply name and address of author to whom proofs should be sent. All other communications should be addressed to the managing editor, or Julie Honig, University of California, Los Angeles, California 90024-1555.

There are page-charges associated with articles appearing in the Pacific Journal of Mathematics. These charges are expected to be paid by the author's University, Government Agency or Company. If the author or authors do not have access to such Institutional support these charges are waived. Single authors will receive 75 free reprints; joint authors will receive a total of 100 free reprints. Additional copies may be obtained at cost in multiples of 50 .

The Pacific Journal of Mathematics (ISSN 0030-8730) is published monthly except for July and August. Regular subscription rate: $\$ 215.00$ a year (10 issues). Special rate: $\$ 107.50$ a year to individual members of supporting institutions.

Subscriptions, orders for numbers issued in the last three calendar years, and changes of address should be sent to Pacific Journal of Mathematics, P.O. Box 4163, Berkeley, CA 94704-0163, U.S.A. Old back numbers obtainable from Kraus Periodicals Co., Route 100, Millwood, NY 10546.

The Pacific Journal of Mathematics at University of California, c/o Department of Mathematics, 981 Evans Hall, Berkeley, CA 94720 (ISSN 0030-8730) is published monthly except for July and August. Second-class postage paid at Berkeley, CA 94704, and additional mailing offices. POSTMASTER: send address changes to Pacific Journal of Mathematics, P.O. Box 4163, Berkeley, CA 94704-0163.

PUBLISHED BY PACIFIC JOURNAL OF MATHEMATICS at University of California, Berkeley, CA 94720, A NON-PROFIT CORPORATION

This publication was typeset using $\mathcal{A} \mathcal{M} S-\mathrm{T}_{\mathrm{E}} \mathrm{X}$, the American Mathematical Society's $\mathrm{T}_{\mathrm{E}} \mathrm{X}$ macro system. Copyright (c) 1994 by Pacific Journal of Mathematics 


\section{PACIFIC JOURNAL OF MATHEMATICS}

Volume $162 \quad$ No. $2 \quad$ February 1994

On the existence of convex classical solutions to multilayer fluid

201 problems in arbitrary space dimensions

ANDREW FRENCH ACKER

Extremal functions and the Chang-Marshall inequality

VALENTIN V. ANDREEV and ALEC LANE MATHESON

Productive polynomials

RICHARD ARENS

On factor representations of discrete rational nilpotent groups and the 261 Plancherel formula

LAWRENCE JAY CORWIN and CAROLYN PFEFFER JOHNSTON

Commutants of Toeplitz operators on the Bergman space

ZELJKO CUCKOVIC

When $L^{1}$ of a vector measure is an AL-space

GUILlERMO P. CURBERA

A convexity theorem for semisimple symmetric spaces

305

KARL-HERMANN NEEB

Ideals of finite codimension in free algebras and the fc-localization

AMNON RosenmanN and ShMUEL Rosset

Dec groups for arbitrarily high exponents

BHARATH Al SETHURAMAN

Errata to: "The set of primes dividing the Lucas numbers has density $2 / 3$ "

JEFFREY C. LAGARIAS 